ORIGINAL ARTICLE

\title{
Persistent high risk HPV infection associated with development of cervical neoplasia in a prospective population study
}

\author{
K S Cuschieri, H A Cubie, M W Whitley, G Gilkison, M J Arends, C Graham, E McGoogan
}

J Clin Pathol 2005;58:946-950. doi: 10.1136/jap.2004.022863

See end of article for authors' affiliations ......................

Correspondence to: Dr K Cuschieri, Specialist Virology Centre, Royal Infirmary of Edinburgh, 51 Little France Crescent, Edinburgh EHI 6 4SA', UK; kate.cuschieri@luht.scot. nhs.uk

Accepted for publication 30 November 2004

\begin{abstract}
Aims: To monitor the association between the course of high risk human papillomavirus (HR-HPV) infection and the development of cervical neoplasia over time, from a baseline of normal cervical cytology. Methods: This paper presents the follow up data from a previous cross sectional analysis. Women from a screening population who had normal cytology and who were HR-HPV positive were recalled after two to three years for cytology and HPV genotyping. The development of cervical neoplasia at follow up was related to the course of HPV infection (clearance, persistence, or sequential infection) and the presence of single or multiple HPV infections at baseline. A comparator control group of women who were HPV and cytologically negative at baseline were selected from the same population.

Results: Twelve cases of dyskaryosis were found in women who were HPV positive at baseline; four were high grade. Only three cases of low grade dyskaryosis were found in the control group. Women with type specific persistent infections were significantly more likely to develop cervical neoplasia than women who cleared the infection $(p=0.0001)$ or were sequentially infected with different types $(p=0.001)$. Women with multiple HPV infections at baseline were no more likely to develop cervical dyskaryosis than those with a single infection.

Conclusions: Type specific persistent HR-HPV infection as monitored by genotyping can identify women at increased risk of cervical neoplasia more accurately than a single or repeated presence/absence HPV test. The cost effectiveness of such an approach should be investigated by an appropriate, large scale costbenefit analysis.
\end{abstract}

1 nfection with high risk human papillomavirus (HR-HPV) is necessary for the development of cervical cancer and its associated precursor, cervical intraepithelial neoplasia. ${ }^{12}$ However, genital HPV infection is common, especially in young women, and frequently shows a transient course. ${ }^{1}$ This reduces the effectiveness of a single HPV detection test in terms of specificity for prediction of progressive disease. A summary of the experts' meeting at the EUROGIN 2003 conference stated that "persistent infection (with HPV) is the single best predictor of cervical cancer". ${ }^{2}$ This conclusion was based on longitudinal studies that monitored the course of HPV infection and its impact on cancer progression. Some of these studies included tests that detect the presence/absence of at least one of a pool of "common" HR-HPV types, such as the Digene hybrid capture ${ }^{\circledR} 2$ test. $^{3}{ }^{4}$ There are benefits to such a test, which include ease of result interpretation and amenability to automation. However, sequential positive results do not identify the persistence of a specific type of HPV, whereas HPV genotyping assays can delineate specific types and are thus more suited to detecting persistent infection. Genotyping can also detect multiple HPV infections, although the clinical usefulness of this diagnosis remains controversial.

"Human papillomavirus genotyping assays can delineate specific types and are thus more suited to detecting persistent infection"

Few studies have compared HPV persistence, as monitored by an HPV genotyping approach, with a presence/absence HPV test over time. ${ }^{5}$ If HPV testing does become incorporated within screening, determining which type of HPV test is the most informative and cost effective is crucial.

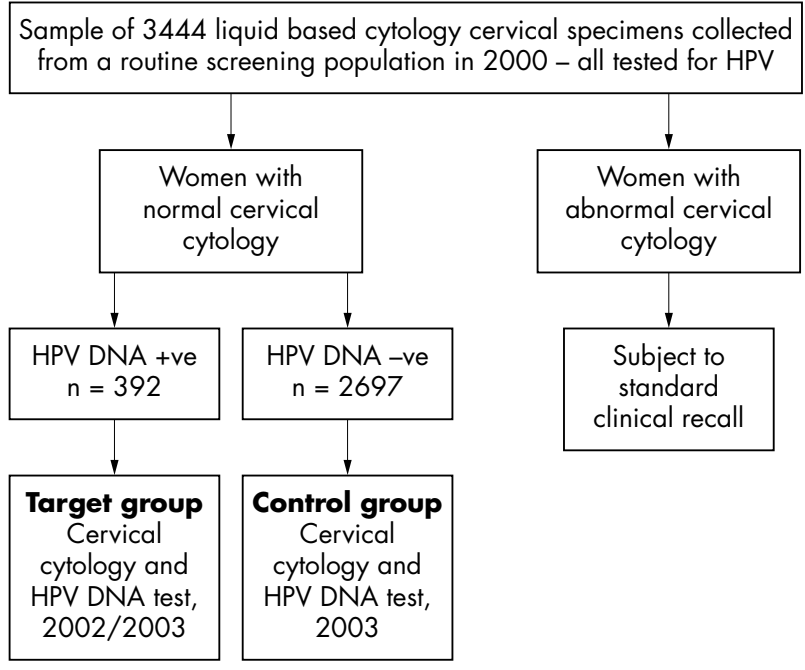

Figure 1 Recruitment of study participants: target and control groups and human papillomavirus (HPV) testing strategy.

We previously reported on a group of 3444 women from a routine cervical screening population in which HPV positivity and prevalence of multiple HR-HPV infections was related to age and grade of cervical neoplasia. ${ }^{7}$ A cohort of 126 women who were HR-HPV positive at baseline with normal cytology (fig 1) agreed to have follow up cytology and HPV genotyping

Abbreviations: HPV, human papillomavirus; HR, high risk; LBC, liquid based cytology 
two to three years later. These samples were analysed for evidence of cervical neoplasia and its relation to three HPV related factors, namely: (1) HR-HPV infection at baseline versus absence, (2) multiple HR-HPV infections at baseline versus single HR-HPV infection, and (3) type specific HRHPV persistence compared with sequential infection by different HPV types and clearance of HPV infection.

\section{MATERIALS AND METHODS}

Recruitment of study participants and recall strategy Women were recruited from the screening population through general practitioner practices across Edinburgh, Scotland, as described previously. ${ }^{1}$ In total, 126 women who were cytology negative and HPV positive in 2000 (baseline) returned for a liquid based cytology (LBC) sample to be taken and HPV testing carried out (mean recall period, 2.2 years). This group of women was termed the "target group" and had a median age of 29.6 years. LBC samples were also obtained from a control group of 99 women from the same population who were negative for cytology and HPV at baseline in 2000, and who consented to repeat cytology and HPV testing at follow up (mean recall, 3.1 years; median age, 37.5 years). Written consent was obtained from all study participants and ethics approval was obtained from the Lothian research ethics committee.

\section{Cervical cytological grading and HPV testing}

Flat layer slides were made by the ThinPrep ${ }^{\circledR}$ procedure. Cervical cytological grading was performed according to British Society for Clinical Cytology guidelines. Nucleic acid extraction of LBC material was performed as described previously. ${ }^{8}$ HPV detection and genotyping were performed by application of $\mathrm{GP} 5+/ 6+$ real time polymerase chain reaction and a reverse line blot assay containing 18 high risk HPV probes and nine low risk HPV probes (Roche Molecular Systems, Alameda, California, USA). ${ }^{9-11}$

\section{Association between HPV status and evidence of cervical neoplasia at follow up}

Analysis of the association between the baseline HPV result and cervical neoplasia follow up at in the target and control groups was performed by the $\chi^{2}$ test, with confidence intervals for differences in proportions. Logistic regression was performed with follow up cervical neoplasia status as the outcome and HPV at baseline (single $v$ multiple HPV infection) and age $(\leqslant 30 v>30)$ as the predictors. Women who had persistent HPV infection (defined as at least one HPV of the same type being present in both baseline and follow up samples) were compared (in terms of number of cases with cervical neoplasia at follow up) with those who were HPV positive at baseline but negative at follow up (clearance of HPV infection) and those who were HPV positive at baseline but who tested positive for different HPV type(s) at follow up (sequential HPV infection). Women were age stratified within these three groups. Fisher's exact test was used to analyse the difference in proportions between
Table 2 Results of human papillomavirus (HPV) genotyping at baseline and follow up in women who had any cervical dyskaryosis detected at follow up

\begin{tabular}{|c|c|c|}
\hline Follow up cytology result & HPV at baseline & HPV at follow up \\
\hline Borderline* $^{*}$ & $x \ddagger$ & 45 \\
\hline Borderline* $^{*}$ & $x \ddagger$ & 39 \\
\hline Borderline* $^{*}$ & 16 & 16 \\
\hline Borderline† & Negative & 35 \\
\hline Borderline† & Negative & $x \ddagger$ \\
\hline Mild* & 56,73 & 56 \\
\hline Mild* & 16,58 & 16,58 \\
\hline Mild* & 33,58 & 33,82 \\
\hline Mild* & $16,52,82,6,42$ & 16,53 \\
\hline Mild* & 31 & Negative \\
\hline Mild* & 59 & $39,56,59$ \\
\hline Mild* & 6 & 73,11 \\
\hline Mild* & 16 & 16,33 \\
\hline Mild* & 16,58 & 16,58 \\
\hline Mild $†$ & Negative & $31,73,53$ \\
\hline Moderate* & 16 & 16 \\
\hline Moderate* & 18 & 18 \\
\hline Severe* & 16 & 16 \\
\hline Severe* & 16 & 16 \\
\hline
\end{tabular}

*Woman within the target group; twoman within the control group; fHPV type that could not be identified by the reverse line blot assay.

women of $\leqslant 30$ versus $>30$ years of age and cervical neoplasia associated with persistence of HPV infection.

\section{RESULTS \\ HPV status at baseline and its relation to cervical neoplasia at follow up}

A total of 16 of 126 women within the target group had detectable cervical neoplasia at follow up: 12 with low grade abnormalities (borderline or mild dyskaryosis) and four with high grade cervical neoplasia (moderate or severe dyskaryosis). In the control group, three women exhibited low grade cervical neoplasia at follow up.

Table 1 shows the baseline HPV status in relation to the cytology result at follow up of women in both the target and control groups. All dyskaryotic grades (borderline, mild, moderate, or severe dyskaryosis) were combined into an "abnormal cytology" category to allow for comparison of baseline HPV positive versus HPV negative status for development of any cervical neoplasia at follow up. Chi square analysis revealed that women were significantly more likely to develop cervical neoplasia if infected with any HRHPV at baseline compared with HPV negative controls $(p=0.010)$. Of the women in the target group, $32.5 \%$ had multiple HR-HPV infections at baseline yet were no more likely to develop abnormalities at follow up than women who were infected with a single type $(p=0.650)$. Logistic regression analysis revealed this finding was consistent when women were subdivided into $\leqslant 30$ and $>30$ years of age. Analysis of the type specific HPV infection detected in women who developed cervical neoplasia showed that persistent infection by either HPV-16 or HPV-18 is associated with high

Table 1 Human papillomavirus (HPV) status and relation to cytological evidence of cervical neoplasia at follow up

\begin{tabular}{lllll}
\hline Follow up cervical cytology result & $\begin{array}{l}\text { HPV -ve at baseline } \\
\text { (control) }\end{array}$ & $\begin{array}{l}\text { HPV +ve at baseline } \\
\text { (target) }\end{array}$ & $\begin{array}{l}\text { Single HPV type at } \\
\text { baseline (target) }\end{array}$ & $\begin{array}{l}\text { Multiple HPV types at } \\
\text { baseline (target) }\end{array}$ \\
\hline Normal & 96 & 110 & 75 & 35 \\
Borderline & 2 & 3 & 3 & 0 \\
Mild & 1 & 9 & 4 & 5 \\
Moderate & 0 & 2 & 1 & 1 \\
Severe & 0 & 2 & 2 & 0 \\
All & 99 & 126 & 85 & 41 \\
\hline
\end{tabular}

HPV status: negative or positive for high risk HPV at baseline, separated into single or multiple high risk HPV infection. 


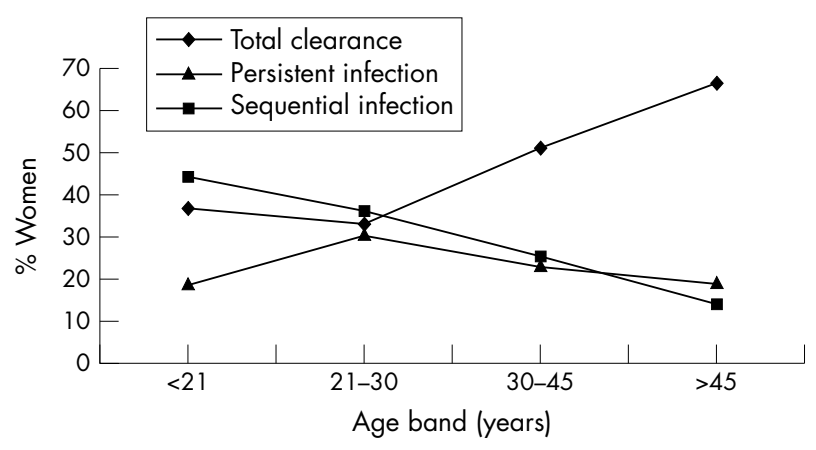

Figure 2 Course of persistent, sequential, and cleared human papillomavirus (HPV) infection according to age.

grade dyskaryosis, whereas a wider range of HPV types was associated with low grade dyskaryosis (table 2 ).

\section{Natural history of HPV infection and its relation to age and development of cervical neoplasia}

In total, 56 of $126(44 \%)$ women in the target group were negative for HPV at follow up, indicating clearance of the HPV infection. In contrast, 70 of 126 (56\%) remained HPV positive, with 29 of 67 being persistently infected (by at least one HR-HPV type) and 38 of 67 testing positive for a different HPV type or types (sequential HPV infection) at follow up. It was not possible to assign the three women who tested positive for unknown HPV types at both time points to the sequential or persistent infection groups, so they were omitted from the analysis. The extent of HPV clearance, persistent infection, and sequential infection was analysed according to age (fig 2; table 3 ).

Sequential HPV infection decreased with age and a peak of persistent HPV infection was found in the 21-30 year olds. Total clearance of HPV was at its lowest in women under 30 years of age, which was reflected in the higher sequential HPV infection levels seen in this age group compared with women over 30 years.

Table 4 shows the course of HPV infection in relation to cytological result at follow up. Of the 29 individuals with persistent HR-HPV infections, 12 went on to develop neoplasia: eight low grade (borderline and mild) and four high grade (moderate and severe) dyskaryosis. Of those with sequential infections, three of 38 developed neoplasia (all low grade) and only one of 56 women who cleared their infection developed a low grade neoplasia. Thus, those who had persistent HR-HPV infection were more likely to develop neoplasia than those who cleared the infection ( $\mathrm{p}=0.0001$; $\chi^{2}$ test) or those who were sequentially infected by different types $\left(\mathrm{p}=0.001 ; \chi^{2}\right.$ test $)$.

Further analysis was performed to assess whether any specific HPV types were more or less likely to persist. Table 5 shows the frequency of HPV types at baseline and the frequency of the same HPV type at follow up. For individuals with multiple HPV infections, each individual HPV type was counted and analysed for persistence.

Analysis of the likelihood of persistence of the individual types could not be performed by formal statistics because our type specific data were to small; however, some HPV types appeared to be more easily eradicated than others-for example, HPV-73 and HPV-51. In addition, comparison of the total HR and total low risk HPV types detected showed that the HR-HPVs (34 of 133) were more likely to form persistent infections than the low risk HPVs (three of 27), with the difference in proportions being $14.5 \% \quad(95 \%$ confidence interval, $0.5 \%$ to $25.4 \%$; $=0.135$; Fishers exact test).

The number of cases of cervical neoplasia was stratified by age and compared with the relevant course of underlying HPV infection (table 6). We found the highest proportion of persistent HR-HPV infection in the 21-30 year olds, although this group did not show an increase in associated cervical neoplasia. Using a binomial test for difference in proportions, no significant differences in the proportions of cervical neoplasia cases (with low and high grade dyskaryosis being assessed cumulatively) were associated with HPV persistence in the under 30 year olds compared with the over 30 year olds. Numbers were too small to perform this analysis with respect to high grade cervical neoplasia exclusively. However, it is notable that of the four women who were $>45$ years old with persistent HR-HPV infection, three developed cervical neoplasia, with two being of high grade.

\section{DISCUSSION}

In our study, the strength of HPV genotyping lay in the identification of clearance, persistence, or sequential infection, rather than the identification of multiple HPV infections. Multiple HR-HPV infections did not constitute a higher risk factor for the development of cervical neoplasia over two to three years compared with single HR-HPV infection. In our previous cross sectional study, we found that multiple HPV infections were more common in young women. ${ }^{7}$ However, no age dependent risk of cervical neoplasia associated with multiple HPV infections was detected in our present study when women under and over 30 were compared. These findings are consistent with the data of Rolón et al. ${ }^{6}$

Our key finding was that women who harboured persistent HR-HPV were significantly more likely to develop cervical neoplasia than those who were sequentially infected by different HR-HPV types or who cleared their infection. This reinforces the elegant work by Kjaer et al, who studied type specific HPV persistence prospectively, and that of Wallin et al, who monitored HPV type specific persistence before the development of cervical cancer..$^{12}$

Although the number of cases of cervical neoplasia was small, it was notable that no women $<30$ years developed high grade cervical neoplasia over the two to three year time frame of our study, despite the highest proportion of persistent HPV infections being seen in the 21-30 year old age group. However, HPV variant analysis was not performed, so it could be that individuals who showed the same

Table 3 Natural history of human papillomavirus (HPV) infection (clearance, persistence, or sequential infection) in women who tested HPV positive at baseline

\begin{tabular}{lllll}
\hline Age at baseline (years) & Number tested & $\begin{array}{l}\text { Total clearance } \\
\text { No. }(\%)\end{array}$ & $\begin{array}{l}\text { Sequential infection } \\
\text { No. }(\%)\end{array}$ & $\begin{array}{l}\text { Persistent infection } \\
\text { No. (\%) }\end{array}$ \\
\hline$<21$ & 27 & $10(37.0)$ & $12(44.4)$ & $5(18.5)$ \\
$21-30$ & 36 & $12(33.3)$ & $13(36.1)$ & $11(30.6)$ \\
$31-45$ & 39 & $20(51.3)$ & $10(25.6)$ & $9(23.1)$ \\
$>45$ & 21 & $14(66.7)$ & $3(14.3)$ & $4(19.0)$ \\
\hline
\end{tabular}


Table 4 Persistent and sequential human papillomavirus (HPV) infections and clearance of HPV infection related to evidence of cervical cytological dyskaryosis at follow up

\begin{tabular}{llccc}
\hline $\begin{array}{l}\text { Follow up cervical cytology result } \\
\text { (total no.) }\end{array}$ & $\begin{array}{l}\text { HPV type specific } \\
\text { persistent infection }\end{array}$ & Sequential HPV infection & $\begin{array}{l}\text { Clearance of HPV } \\
\text { infection }\end{array}$ \\
\hline Negative (1 10) & 17 & 35 & 55 & 3 \\
Borderline (3) & 1 & 2 & 0 & 0 \\
Mild (9) & 7 & 1 & 1 & 0 \\
Moderate (2) & 2 & 0 & 0 & 0 \\
Severe (2) & 2 & 0 & 0 & 0 \\
All & 29 & 38 & 56 & 3
\end{tabular}

*3 specimens were HPV positive at baseline and follow up but could not be assigned to either HPV persistent or sequential infections because the specific HPV type could not be determined; therefore, they were excluded from subsequent analysis.

HR-HPV type at baseline and follow up were, in fact, sequentially infected with the same type. This phenomenon could be particularly pronounced in the $21-30$ year olds who (as seen in our study) experience a high frequency of sequential HPV infections, probably associated with greater sexual activity. ${ }^{14}$

All cases of high grade cervical neoplasia were seen in women $>30$ years and all were associated with type specific persistent HR-HPV infection. In particular, of the 21 women $>45$ years of age, four harboured persistent HPV infection, and three of these developed neoplasia (two high grade). In addition, there were no cases of "any grade" of cervical neoplasia associated with a non-persistent HPV infection in the $>45$ year olds. Repeated HPV genotyping could be used to identify persistent HPV infection and perhaps this approach would be most useful in cervical screening programmes in women $>30 .{ }^{15}$ Certainly, the high prevalence of HPV in young women and the benign nature of most of their abnormalities have influenced screening policies in other countries. For example, in the Netherlands and

\begin{tabular}{|c|c|c|c|}
\hline HPV type & Baseline & Follow up & \% Persistence \\
\hline \multicolumn{4}{|l|}{ HR-HPV type } \\
\hline 16 & 33 & 16 & 48.5 \\
\hline 18 & 10 & 2 & 20.0 \\
\hline 31 & 10 & 1 & 10.0 \\
\hline 33 & 5 & 3 & 60.0 \\
\hline 35 & 1 & 0 & 0 \\
\hline 39 & 1 & 0 & 0 \\
\hline 45 & 7 & 1 & 14.3 \\
\hline 51 & 12 & 0 & 0 \\
\hline 52 & 6 & 1 & 16.7 \\
\hline 53 & 9 & 1 & 11.1 \\
\hline 56 & 7 & 2 & 28.6 \\
\hline 58 & 6 & 2 & 33.3 \\
\hline 59 & 7 & 4 & 57.1 \\
\hline 66 & 9 & 1 & 11.1 \\
\hline 68 & 2 & 0 & 0 \\
\hline 73 & 7 & 0 & 0 \\
\hline 82 & 1 & 0 & 0 \\
\hline $\begin{array}{l}\text { Total HR-HPV } \\
\text { R-HPV }\end{array}$ & 133 & 34 & $25.6 \%$ \\
\hline 6 & 5 & 0 & 0 \\
\hline 11 & 2 & 0 & 0 \\
\hline 40 & 2 & 0 & 0 \\
\hline 42 & 9 & 2 & 22.2 \\
\hline 54 & 2 & 0 & 0 \\
\hline 55 & 2 & 1 & 50.0 \\
\hline 57 & 1 & 0 & 0 \\
\hline 83 & 1 & 0 & 0 \\
\hline 84 & 3 & 0 & 0 \\
\hline Total LR-HPV & 27 & 3 & $11.1 \%$ \\
\hline
\end{tabular}

Human papillomavirus (HPV) types within mixed infections are described separately. The table is subdivided into high risk (HR) and low risk (LR) HPV types and their totals are calculated.
Norway, cervical screening is not initiated until age 30 and the Food and Drug Administration in the USA has recommended HPV testing in combination with cytology for primary screening in women $>30$ years. ${ }^{16}$

\section{"A single human papillomavirus (HPV) test that can detect or predict HPV persistence would be extremely useful within a screening context"}

Extrapolation of our results suggests that repeated genotyping might be more specific for identifying those women at risk of cervical neoplasia compared with repeated testing using a presence/absence HPV test. In our small study, over $40 \%$ of women with HR-HPV persistence developed cervical neoplasia at follow up compared with $8 \%$ who were sequentially infected, and high grade dyskaryosis was not seen in this last group.

Within the literature there is no clear consensus definition of persistent HPV infection, so that if repeated genotyping is to be used to identify persistence, an appropriate time between tests must be defined. In addition, repeated testing would have cost and laboratory implications. We are not aware of an algorithm that has factored in identification of type specific persistent HPV infection and impact on disease detection and associated referrals.

A single HPV test that can detect or predict HPV persistence would be extremely useful within a screening context. RNA based approaches may be suitable, in that transcribed HPV oncogenes directly relevant to neoplasia are targeted rather than the structural gene Ll, the classic target for DNA based assays. We have reported the prognostic value of a "one off" RNA based test for E6 and E7 mRNA transcripts. ${ }^{17} \mathrm{HPV}$ viral load measurement may also be predictive of future cervical neoplasia and a linear association between increasing grade of cervical intraepithelial neoplasia lesions and HPV-16 viral load has been noted. ${ }^{418} 19$ However, systems for measuring cumulative viral load may overestimate type specific loads within multiple HPV infections. ${ }^{19}$ Moreover, it has been suggested that there may be type specific differences in the clinical usefulness of viral load. ${ }^{19}$ In addition, technical limitations associated with multiplexing HPV type specific quantitative assays on real time polymerase chain reaction platforms make this approach, at least at present, unsuitable for detecting the diverse range of HR-HPV types within screening populations.

This prospective population based study in Scotland has shown that women infected with multiple HPV types at baseline were no more likely to develop cervical dyskaryosis after two to three years than those with a single HR-HPV infection. Women who had persistent HR-HPV infections were at greater risk of developing cervical neoplasia compared with those who cleared HPV $(p=0.0001)$ or those who were sequentially infected with different HPV types $(p=0.001)$. All high grade cervical neoplasms were 
Table 6 Age stratification of women who developed cervical neoplasia on cytological examination in relation to the natural history of HPV infection

\begin{tabular}{llllll}
\hline Age (years) & CN cases & $\begin{array}{l}\text { No. with persistent } \\
\text { HPV }\end{array}$ & $\begin{array}{l}\text { CN cases associated with HPV } \\
\text { persistence }\end{array}$ & $\begin{array}{l}\text { No. of *non-persistent } \\
\text { HPV infections }\end{array}$ & $\begin{array}{l}\text { CN cases not associated with } \\
\text { HPV persistence }\end{array}$ \\
\hline$<21$ & $4(14.8)$ & $5(18.5)$ & 3 (low grade) & $22(81.5)$ & 1 low grade \\
$21-30$ & $4(11.1)$ & $11(30.6)$ & 3 (low grade) & $25(69.4)$ & 1 low grade \\
$31-45$ & $5(12.8)$ & $9(23.1)$ & 3 (2 high grade, 1 low grade) & $30(76.9)$ & 2 low grade \\
$>45$ & $3(14.3)$ & $4(19.0)$ & $3(2$ high grade, 1 low grade) & $17(81.0)$ & 0 \\
Total & 16 & 29 & 12 & 94 & 4
\end{tabular}

Figures in parenthesis are the percentage of the age band tested.

*Non-persistent, baseline HPV infections cleared plus sequential HPV infections.

$\mathrm{CN}$, cervical neoplasia; HPV, human papillomavirus.

\section{Take home messages}

- Women infected with multiple human papillomavirus (HPV) types were no more likely to develop cervical dyskaryosis after two to three years than those with a single high risk (HR) HPV infection

- Women with persistent HR-HPV infections were at greater risk of developing cervical neoplasia than those who cleared HPV or were sequentially infected with different HPV types

- All high grade cervical neoplasms were detected in women over 30 years and all were associated with persistent HR-HPV infection

- Thus, repeated HPV genotyping could be used to monitor type specific persistent HR-HPV infection as a means of identifying women at increased risk of cervical neoplasia, and might be more accurate than either a one off HPV test or a repeated presence/ absence HPV test

- The cost effectiveness of such an approach should be investigated by an appropriate, large scale costbenefit analysis

detected in women over 30 years and all were associated with persistent HR-HPV infection. Thus, we conclude that repeated HPV genotyping could be used to monitor type specific persistent HR-HPV infection as a means of identifying women at increased risk of cervical neoplasia. This approach could prove more accurate than either a one off HPV test or a repeated presence-absence HPV test. The cost effectiveness of such an approach should be investigated by an appropriate, large scale cost-benefit analysis.

\section{ACKNOWLEDGEMENTS}

The authors wish to thank Roche Molecular Systems for the supply of the HPV genotyping test materials. We are also grateful to C Moore and $\mathrm{F}$ Murray Zmijewski for performing specimen preparation procedures and J Bingham for assistance with collating cytopathology information. This work was funded by the Chief Scientist Office of the Scottish Executive, grant no $\mathrm{CZB} / 4 / 33$, and ethical approval was granted by Lothian research ethics committee (reference LREC/ 2001/1/25).

\section{Authors' affiliations}

K S Cuschieri, H A Cubie, G Gilkison E McGoogan, Royal Infirmary of Edinburgh, 51 Little France Crescent, Edinburgh EH16 4SA, UK M W Whitley, St Triduanas Medical Practice, 54 Moira Park, Edinburgh EH16 4SA, UK
M J Arends, University of Cambridge Pathology Department, Addenbrooke's Hospital, Hills Road, Cambridge CB2 2QQ, UK C Graham, The Epidemiology and Statistics Core, Wellcome Trust Clinical Research Facility, University of Edinburgh, Western General Hospital, Edinburgh EH4 2XU, UK

\section{REFERENCES}

1 Ho GY, Bierman R, Beardsley L, et al. Natural history of cervicovaginal papillomavirus infection in young women. N Engl J Med 1998;338:423-8.

2 Muñoz N, Bosch FX, de Sanjose S, et al. Epidemiologic classification of human papillomavirus types associated with cervical cancer. N Engl J Med 2003;348:518-27.

3 Dalstien V, Riethmuller D, Pretet JL, et al. Persistence and load of high-risk HPV are predictors for development of high-grade cervical lesions: a longitudinal French cohort study. Int J Cancer 2003;106:396-403.

4 Bory JP, Cucherousset J, Lorenzato M, et al. Recurrent human papillomavirus infection detected with the hybrid capture 2 assay selects women with normal cervical smears at risk for developing low grade cervical lesions: a longitudinal study of 3091 women. Int J Cancer 2002;102:519-25.

5 Fife $\mathrm{KH}$, Craner HM, Schroeder JM, et al. Detection of multiple human papillomavirus types in the lower genital tract correlates with cervical dysplasia. J Med Virol 2001;64:550-9.

6 Rolón PA, Smith JS, Muñoz N, et al. Human papillomavirus infection and invasive cervical cancer in Paraguay. Int J Cancer 2000;85:486-91.

7 Cuschieri KS, Cubie HA, Whitley MW, et al. Multiple high risk HPV infections are common in cervical neoplasia and young women in a cervical screening population. J Clin Pathol 2004;57:68-72.

8 Cuschieri KS, Seagar AL, Moore C, et al. Development of an automated extraction procedure for detection of human papillomavirus DNA in liquid based cytology samples. J Virol Methods 2003;107:107-13.

9 Cubie HA, Seagar AL, McGoogan E, et al. Rapid real time PCR to distinguish between high-risk human papillomavirus types 16 and 18. Mol Pathol $2001 ; 54: 24-9$

10 Coutlee F, Gravitt P, Kornegay J, et al. Use of PGMY primers in L1 consensus PCR improves detection of human papillomavirus DNA in genital samples. J Clin Microbiol 2002:40:902-7.

11 Gravitt PE, Peyton CL, Apple RJ, et al. Genotyping of 27 human papillomavirus types by using L1 consensus PCR products by a single hybridization, reverse line blot detection method. J Clin Microbiol 1998;36:3020-7.

12 Kjaer SK, van den Brule AJC, Gerson P, et al. Type specific persistence of high risk human papillomavirus (HPV) as indicator of high grade cervical squamous intraepithelial lesions in young women: population based prospective follow up study. BMJ 2002;325:572-6

13 Wallin KL, Wiklund F, Angstom T, et al. Type-specific persistence of human papillomavirus DNA before the development of cervical cancer. N Engl J Med 1999;341:1633-8

14 Mayrand MH, Coutlee F, Hankins C, et al. Detection of human papillomavirus type 16 DNA in consecutive genital samples does not always represent persistent infection as determined by molecular variant analysis. J Clin Microbiol 2000;38:3388-93.

15 Monsonego J, Bosch FX, Cuorsaget $\mathrm{P}$, et al. Cervical cancer control priorities and new directions. Int J Cancer 2004; 108:329-33.

16 Wright TC, Schiffman M, Solomon D, et al. Interim guidance for the use of human papillomavirus DNA testing as an adjunct to cervical cytology for screening. Am Coll Obstet Gynecol 2004;103:304-9.

17 Cuschieri KS, Whitley MJ, Cubie HA, et al. Human papillomavirus type specific DNA and RNA persistence-implications for cervical disease progression and monitoring. J Med Virol 2004;73:65-70.

18 Ylitalo N, Sorenson P, Josefsson AM, et al. Consistent high viral load of human papillomavirus 16 and risk of cervical cancer in situ: a nested case control study. Lancet 2000;355:2194-8.

19 Gravitt PE, Burk RD, Lorincz A, et al. A comparison between real-time polymerase chain reaction and hybrid capture 2 for human papillomavirus quantitation. Cancer Epidemiol Biomarkers Prev 2003;12:477-84. 